\title{
Nanoscale
}

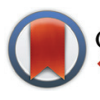

CrossMark \& click for updates

Cite this: Nanoscale, 2014, 6, 13535

Received 22nd July 2014,

Accepted 17th September 2014

DOI: $10.1039 / c 4 n r 04151 h$

www.rsc.org/nanoscale

\section{Metallic nanowire networks: effects of thermal annealing on electrical resistance}

\author{
D. P. Langley, ${ }^{a, b}$ M. Lagrange, ${ }^{a}$ G. Giusti, ${ }^{a}$ C. Jiménez, ${ }^{a}$ Y. Bréchet, ${ }^{c}$ N. D. Nguyen ${ }^{b}$ \\ and D. Bellet*a
}

\begin{abstract}
Metallic nanowire networks have huge potential in devices requiring transparent electrodes. This article describes how the electrical resistance of metal nanowire networks evolve under thermal annealing. Understanding the behavior of such films is crucial for the optimization of transparent electrodes which find many applications. An in-depth investigation of silver nanowire networks under different annealing conditions provides a case study demonstrating that several mechanisms, namely local sintering and desorption of organic residues, are responsible for the reduction of the systems electrical resistance. Optimization of the annealing led to specimens with transmittance of $90 \%$ (at $550 \mathrm{~nm}$ ) and sheet resistance of $9.5 \Omega \mathrm{sq}^{-1}$. Quantized steps in resistance were observed and a model is proposed which provides good agreement with the experimental results. In terms of thermal behavior, we demonstrate that there is a maximum thermal budget that these electrodes can tolerate due to spheroidization of the nanowires. This budget is determined by two main factors: the thermal loading and the wire diameter. This result enables the fabrication and optimization of transparent metal nanowire electrodes for solar cells, organic electronics and flexible displays.
\end{abstract}

\section{Introduction}

Metallic nanowires (NWs) have recently received increasing interest due to their potential application as interconnects and as essential components in nanoelectronics. ${ }^{1}$ Metallic NW networks are also promising candidates to be used as a transparent conductive material for many industrial purposes. Their physical and chemical properties differ from the properties of the bulk material and are strongly related to both the size and structure of the NWs themselves. When used as a network, their characteristics, such as wire diameter, density of wires in the network and junction resistance, have a significant impact on the physical properties of the whole. ${ }^{2}$

When working with metallic nanowires for transparent electrode applications the main goal is to obtain an electrode which has the lowest electrical conductivity for a given optical transmission.

Optimizing the electrical resistance and optical transmission of nanowire networks involves tuning several key parameters including the experimental conditions of deposition, wire geometry, ${ }^{3}$ network density and post-deposition treatments

\footnotetext{
${ }^{a}$ Univ. Grenoble Alpes, LMGP, F-38000 Grenoble, France. E-mail: daniel.bellet@grenoble-inp.fr

${ }^{b}$ Laboratoire de Physique des Solides, Interfaces et Nanostructures; Département de Physique, Université de Liège, Allée du 6 Août 17, B-4000 Liège, Belgium

${ }^{c}$ Univ. Grenoble Alpes, SIMAP, F-38000 Grenoble, France
}

such as mechanical pressing, ${ }^{4}$ light-induced plasmonic nanowelding ${ }^{5-7}$ or thermal annealing. ${ }^{8-10}$ All of these postdeposition treatments have been shown to decrease the electrical resistance. However, thermal annealing has not yet been the subject of a detailed investigation. The impact of thermal annealing on the electrical resistance of metallic NW networks is dominated by desorption of organic residues and local sintering at the junction between neighbouring nanowires, ${ }^{8,10-12}$ both of which lead to a significantly lower contact resistance. This is a clear asset of metallic NW network when compared with carbon nanotubes which suffer from high junction resistance between nanotubes. ${ }^{13}$

Although thermal annealing can lower the electrical resistance of metallic nanowire networks, instability of the nanowires upon an excessive heating results in a critical limit of the thermal budget applied on the system, which requires dedicated analysis. ${ }^{14}$ Cylindrical metallic nanowires are known to be energetically unstable and can fragment at temperatures significantly lower than their melting point. ${ }^{15-18}$ This is the result of a morphological instability, called the Rayleigh instability, and is a critical issue for the long-term reliability of micro- and nanoelectronic devices. These thermal instabilities place limitations on the thermal budget that an electrode may undergo during device fabrication. For example the use of metal NW electrodes in $\mathrm{TiO}_{2}$ Dye Sensitized Solar Cells (DSSCs) may be restricted due to the need to sinter the $\mathrm{TiO}_{2}$ nanoparticle layers for 1 hour at $450{ }^{\circ} \mathrm{C} .{ }^{19,20}$ It has been suggested recently that a second instabil- 
ity may also be observed due to Joule heating when metallic nanowire networks are used with current densities similar to those found in a solar cell of $\approx 10 \%$ efficiency, leading to electrode failure in a matter of days. ${ }^{21}$ Such instabilities and the mechanisms leading to them need to be understood to enable the efficient integration of metallic NW networks in real devices.

Due to their promising electrical and optical properties, silver nanowires (AgNWs) have been intensively investigated for use as a transparent conductive material. ${ }^{2,5,22-24}$ Such networks have been applied to several different types of devices, including: solar cells, ${ }^{25,26}$ flexible light-emitting devices and displays, ${ }^{27}$ stretchable electrodes ${ }^{28}$ and flexible transparent thin film heaters. ${ }^{29,30}$

In this work, we studied the effects of thermal annealing on the electrical properties of AgNW networks. The main goal is to better understand their behaviour and to optimize the electrical properties of the annealed network. Since several physical mechanisms are at play, different types of annealing have been employed (isothermal, thermal ramps and pre-annealing) in order to disentangle the possible causes responsible for the variations of the electrical resistance observed in situ.

\section{Results}

\subsection{Thermal annealing}

The most direct way to probe the effect of thermal annealing on the electrical resistance of AgNWs is to measure their resistance in situ during a thermal ramp in air. The evolution of the electrical resistance of an AgNW network versus temperature during a thermal ramp at $15{ }^{\circ} \mathrm{C} \min ^{-1}$ in air is shown in Fig. 1a. The electrical resistance varies by several orders of magnitude and clearly exhibits a minimum. Several mechanisms are responsible for the observed variations in resistance as a function of temperature.

This type of annealing, with $T(t)=T_{0}+\dot{T} \cdot t$, where $T(t)$ is the temperature at time $t, T_{0}$ is the initial temperature and $\dot{T}$ is the heating rate, is classically used for the quantitative determination of the desorption activation energy. ${ }^{31}$ At low temperatures $\left(50-125^{\circ} \mathrm{C}\right.$ ) several sudden drops of resistance $R(t, T)$ can be observed in Fig. 1a and 1f (both within the highlighted frames) and will be discussed in detail below. In Fig. 1b a SEM image of an as-deposited nanowire network is displayed. It can be seen that the nanowires are coated with a carbonaceous shell which is approximately $30 \mathrm{~nm}$ thick. This shell is a combination of polyvinylpyrrolidone (PVP) from the nanowire synthesis $^{32}$ and organic residues from the solution based deposition. The removal of these organic contaminants and/or residual isopropyl alcohol (IPA) decreases the electrical resistance of the contact between NWs. The reduced resistance is associated with the formation of efficient conduction pathways through the network.

At higher temperatures $\left(125-170{ }^{\circ} \mathrm{C}\right) R(t, T)$ continues to decrease with increasing temperature and becomes a continuous process which resembles an exponential decay. This can be assigned to the continued desorption of organic residues
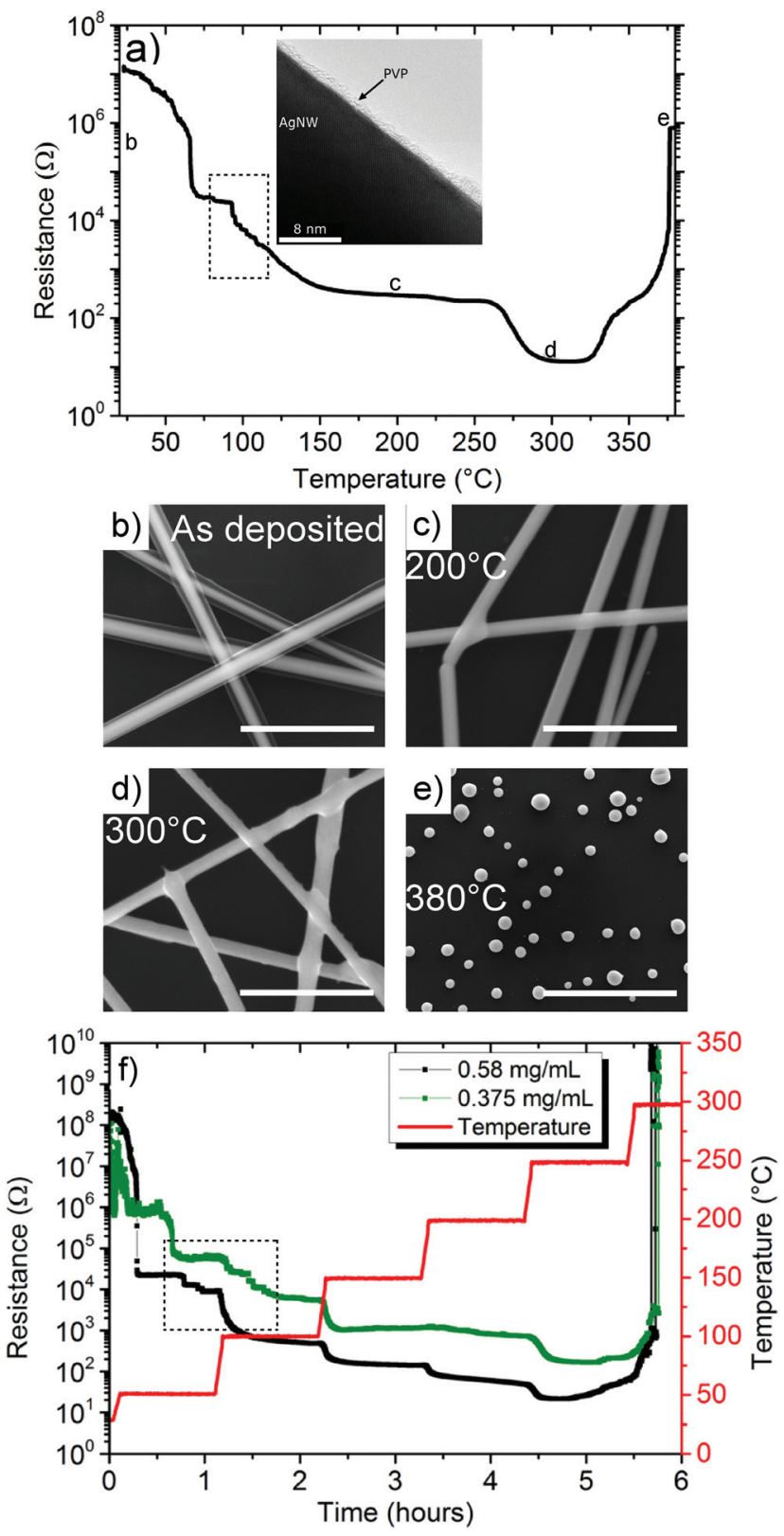

Fig. 1 (a) Evolution of the electrical resistance of a $\mathrm{Ag}$ nanowire network during a continuous thermal ramp of $15^{\circ} \mathrm{C} \mathrm{min}^{-1}$ from room temperature (associated with deposition solution of $0.75 \mathrm{mg} \mathrm{mL}^{-1}$ and an areal mass density of $\approx 105 \mathrm{mg} \mathrm{m}^{-2}$ ). The inset image is a TEM micrograph of a silver nanowire showing residual PVP from synthesis. (b-e) Scanning electron microscope (SEM) images of: (b) as-deposited sample and of specimen annealed for 10 minutes at different temperatures: (c) $200{ }^{\circ} \mathrm{C}$, first occurrence of observable sintering; (d) $300{ }^{\circ} \mathrm{C}$ all junctions are sintered; (e) $380^{\circ} \mathrm{C}$ complete spheroidization of the network. Image (e) is at a lower magnification than the others to demonstrate that although the nanowires are completely spheroidized the resultant nanoparticles are still aligned at the original position of the wires. The scale bars in images (b), (c) and (d) are $1 \mu \mathrm{m}$ whereas that of image (e) is $4 \mu \mathrm{m}$. (f) Resistance response of two specimens to a series of increasing isothermal anneals (associated with deposition solutions of $0.58 \mathrm{mg} \mathrm{mL}^{-1}$ and $0.375 \mathrm{mg} \mathrm{mL}^{-1}$ resulting in areal mass densities of $\approx 81 \mathrm{mg} \mathrm{m}^{-2}$ and $\approx 53 \mathrm{mg} \mathrm{m}^{-2}$, respectively). All data shown in Fig. 1 were obtained using AgNW for which average wire diameter was $105 \mathrm{~nm}$ and average wire length was $37.3 \mu \mathrm{m}$. 
and the polymer coating (PVP) from the AgNWs. Fig. 1c exhibits a SEM image of a specimen annealed at $200{ }^{\circ} \mathrm{C}$ for 10 minutes: it is clearly visible that the majority of the carbonaceous film has been removed, however the thin film $(\sim 2.5 \mathrm{~nm}$ see Fig. 1a inset) of PVP remains on the surface. At $\approx 170{ }^{\circ} \mathrm{C}$ the rate of reduction in $R(t, T)$ slows down as the desorption of organic components tails off due to the decreased concentration of remaining isopropanol and other organic residues. Above $200{ }^{\circ} \mathrm{C}$ local sintering begins at NW junctions, as revealed by the SEM picture shown in Fig. 1c. The rate of sintering is slow at first due to remaining PVP, thermo gravimetric analysis revealed that there is an exothermic reaction occurring between $250{ }^{\circ} \mathrm{C}$ and $270{ }^{\circ} \mathrm{C}$. This result is similar to that found previously by Lee et $a .^{12}$ and was assigned to the degradation of PVP. Above $270{ }^{\circ} \mathrm{C}$ sintering occurs more rapidly resulting in a continued drop in resistance. Again, this takes the form of an exponential function. For this temperature range, as the population of unsintered junctions decreases the rate of junction sintering decreases. A minimum in $R(t, T)$ is observed from $300-320{ }^{\circ} \mathrm{C}$. For higher temperatures $R(t, T)$ increases sharply. This is attributed to the onset of a morphological instability which results in spheroidization of the nanowires as observed in SEM image of Fig. 1e. While thermal annealing has a significant impact on the electrical resistance of AgNW networks (with decrease of several orders of magnitude) the optical transmittance remains identical within experimental errors.

Since electrical properties for a given thermal equilibrium state and kinetic effects are intertwined, it is difficult to determine the exact temperature ranges over which the above-mentioned stages occur from the results shown in Fig. 1a (temperature ramp). However, observation of Fig. 1a-e leads to a general trend of the effect of temperature on silver nanowire networks, which can be described by three main stages:

Reduction. Initially the resistance drops as the residual solvents and PVP are removed from the network. A second reduction phase is observed due to the onset of local sintering at the junctions.

Stabilisation. As the number of unsintered junctions decreases the electrical resistance reduction rate slows down and the resistance value stabilizes briefly, this is associated with the minimum resistance.

Spheroidisation. Morphological instabilities of the nanowires occur and the resistance increases rapidly as the NW network degrades into separated spheres and loses its percolative nature.

Experiments with in situ monitoring of the resistance variation using a thermal ramp provide a nice overview of the thermal annealing effects. However, during a thermal ramp multiple processes may occur simultaneously as they have not had enough time to reach completion before a second mechanism starts. One method to shed light on the thermal annealing effects is thus to consider isothermal annealing with plateaus at various temperatures, as reported in Fig. 1f. The same 3 stages seen in Fig. 1a are observed for the isothermal stepped annealing process depicted in Fig. 1f. Due to the separation of kinetics and activation temperature the mechanisms at play occur at different temperature ranges compared to the ramped annealing shown in Fig. 1a.

Fig. 1f also exhibits sharp drops in the specimen resistance at low temperature $\left(\leq 100{ }^{\circ} \mathrm{C}\right)$ again a detailed discussion of this behavior is provided in section 2.2. At $100{ }^{\circ} \mathrm{C}$ and above, $R(t, T)$ exhibits an initial rapid decrease for each temperature plateau lower than $250{ }^{\circ} \mathrm{C}$. At $250{ }^{\circ} \mathrm{C}$ the resistance plateaus and for a period of several minutes remains constant before $R$ increases due to the onset of the morphological instability of the AgNWs. This then gives indication of both the optimized temperature where the electrical resistance of the network can be minimized through balancing the sintering effects and thermal stability of the AgNWs. The denser network exhibits a lower electrical resistance as a result of the increased number of conduction pathways and at the expense of a lower optical transparency. The network density results in a tradeoff between high optical transparency (obtained for sparse networks) and efficient electrical conductivity (associated with a high NW density). ${ }^{22}$

\subsection{Quantized activation of percolation}

The drops of electrical resistance observed in both Fig. 1a and 1f can be understood in the following way; they correspond to the creation of the first efficient pathways through the network composed of AgNW and junctions between them. The junction resistances are high before thermal annealing and decrease as a function of time when the temperature is increased, as shown by Fig. 1a and 1f. For all specimens studied the resistance drops occur at a resistance of several thousands of ohms, which is of the same order of magnitude as the resistance of a long hypothetical NW crossing the whole network. This estimate is based on Ohm's law applied on an efficient percolating path (EPP) which is a cylinder of length $L$ (in this case the width of the whole network $\approx 7 \mathrm{~mm}$ long), cross-sectional area $S$ and electrical resistivity $\rho$. The associated electrical resistance $R_{\mathrm{EPP}}$ can be written as:

$$
R_{\mathrm{EPP}}=\frac{K \rho L}{S}
$$

where $K$ is a constant associated with the actual path length of the percolating path (which is longer than the network width due to the tortuous nature of percolating paths as schematically shown by Fig. 2a), and therefore on the density of the network, as well as the electrical resistance of the junctions which act as series resistances along the conduction pathway. Therefore, $K$ is expected to be larger than one.

The validity of using silver bulk resistivity $\left(1.59 \times 10^{-8} \Omega \mathrm{m}\right)$ for nanowires of this size is questionable as the wire diameter is approaching the mean free path of electrons in bulk silver. The reduced diameter results in a significant increase in the impact of surface scattering on the resistivity of the wires. It has been demonstrated that the resistivity of nanowires is dependent on the wire diameter and increases above that of the bulk significantly. ${ }^{33}$ In the case of AgNWs with a diameter $100 \mathrm{~nm}$, Bid et al. found the resistivity to be $2.78 \times 10^{-8} \Omega \mathrm{m} .^{33}$ 

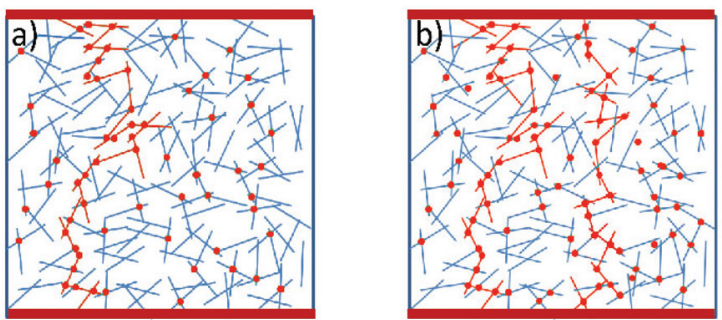

c)
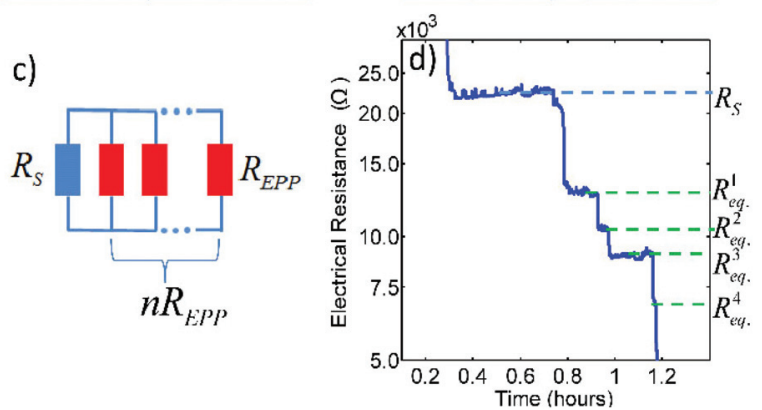

Fig. 2 (a) Schematic representation of a NW network; under thermal annealing, some of the junctions become efficient (indicated by red dots). When a sufficient number of junctions are activated, an efficient percolating pathway forms between the top and bottom electrodes. (b) As heating continues, additional efficient pathways form. (c) The pathways can be considered as $n$ resistances $R_{\text {EPP }}$ in parallel with an initial system resistance $R_{\mathrm{S}}$. (d) Experimental data exhibiting plateaus in resistance which can be assigned as equivalent resistances $R_{\text {eq }}^{n}$ which are the parallel sum of $R_{\mathrm{S}}$ and $n$ resistances $R_{\mathrm{EPP}}$, where $\mathrm{n}$ is an integer which increases as thermal annealing proceeds.

Considering the NW diameter (105 $\mathrm{nm}$ on average), $R_{\mathrm{EPP}}$ is on the order of a few thousand ohms, in good agreement with the steps in resistance observed at low temperature in Fig. 1a and 1f. This first efficient percolating pathway (EPP) (represented schematically in Fig. 2a by thicker red NWs) exhibits an electrical resistance, named $R_{\mathrm{EPP}}$, which should contribute in parallel to the resistance of the initial network, noted $R_{\mathrm{S}}$.

A second EPP appears slightly later, as shown in Fig. 2b) and again acts as an additional parallel resistance $R_{\mathrm{EPP}}$, which leads to another drop of $R$ as shown experimental by Fig. 1a and 1f. Therefore the simplest model defines the electrical resistance $R_{\text {eq }}^{n}$ of the network after the $n^{\text {th }}$ resistance drop as the equivalent resistance consisting of $R_{\mathrm{S}}$ in parallel with $n$ electrical resistances $R_{\mathrm{EPP}}$ (as shown in Fig. 2c), leading to the following expression:

$$
\frac{1}{R_{\mathrm{eq}}^{n}}=\frac{1}{R_{\mathrm{S}}}+\frac{n}{R_{\mathrm{EPP}}}
$$

The experimental values of $R_{\mathrm{eq}}$ are schematically shown on Fig. 2d). $R_{\mathrm{S}}$ can be defined by the experimental resistance before the appearance of the first drop. $R_{\mathrm{EPP}}$ can be determined by eqn (1) where all parameters are known except $K$ which remains the only fitting parameter of the model and is kept constant for all resistance drops observed on a given specimen. The regions which exhibit steps in the resistance from Fig. 1a and 1f are enlarged and compared in Fig. 3a and
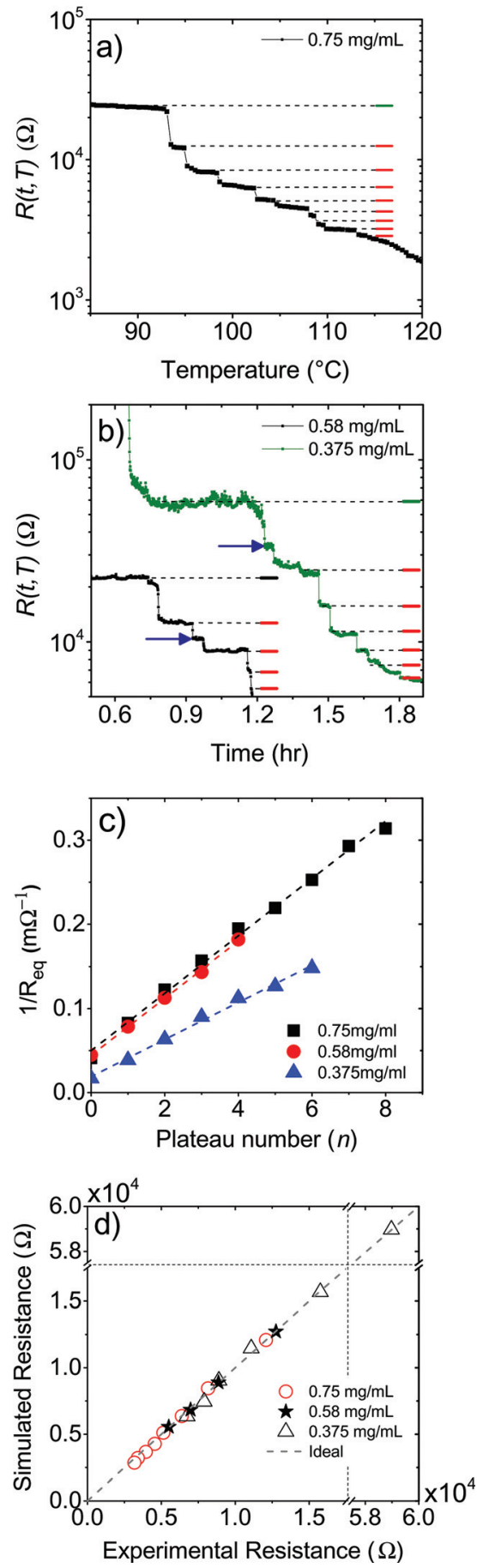

Fig. 3 (a) and (b) Experimental electrical resistance drops observed during thermal annealing (continuous line) for samples of different densities and comparison with the proposed model (dashed lines). Data shown in (a) corresponds to the highlighted section of Fig. 1a (i.e. during a thermal ramp) while data in (b) corresponds to the highlighted section of Fig. If (along a temperature plateau). Arrows in the plot indicate plateaus which were identified as partial percolating pathways. (c) Plot of $1 / R_{\text {eq }}$ as a function of plateau number $n$ extracted from the experimental plateaus of the curves in Fig. $3 a$ and $3 b$ (dashed lines are a linear fit based on eqn (2)). (d) Comparison of the predicted values to experimental electrical resistances, the dashed line represents a slope of one indicating a perfect correlation. The used $K$ values were $1.15,1.31$ and 1.91 associated with deposited solution concentration of $0.75,0.58$ and $0.375 \mathrm{mg} \mathrm{mL}^{-1}$, respectively. 
$3 \mathrm{~b}$ to the values calculated by fitting $K$ using eqn (1) and (2) (shown as solid lines to the right of the experimental data).

Despite the simplicity of the model, the agreement between the experimental data and the calculated values is excellent. From Fig. 3c it is clear that the resistance is described well by eqn (2) and that the fitting parameter $K$ for eqn (1) can be calculated. $R_{\mathrm{S}}$ and $R_{\mathrm{EPP}}$ values were determined from a linear fit of the experimental data (see Fig. 3c) according to eqn (2). The $R_{\mathrm{S}}$ values obtained from these fits correspond well to those observed experimentally, while $R_{\mathrm{EPP}}$ values are larger than $\rho L / S$ in agreement with eqn (1). The latter enables calculation of the $K$ values which are $1.15,1.31$ and 1.91 for specimens associated with solution concentration of $0.75,0.58$ and $0.375 \mathrm{mg}$ $\mathrm{mL}^{-1}$, respectively.

In Fig. 3d a comparison is given between experimental data and values calculated with eqn (1) and (2), showing excellent agreement with a $K$ value varying between 1.15 and 1.91 as the network density decreases. It is not surprising that the value of $K$ increases as the density of the network decreases, as $K$ is a proportionality factor relating to the actual length of the individual conduction pathways. In a dense network there are many more junctions per unit area and the probability of finding a direct path is much higher. As the network density decreases the path becomes more tortuous thus increasing the effective distance between electrodes, and hence the value of $K$.

As with all models there are simplifications that must be made, the proposed model assumes that all pathways are independent and electrically connected in parallel. In reality the system can be more complicated and partial pathways and series connections between pathways may also appear (a schematic way to imagine such a pathway is to consider a $\mathrm{Y}$ shape for the partial pathways and a $\mathrm{H}$ shape for the series connections between paths). Examples can be seen in Fig. $3 \mathrm{~b}$ where the plateaus indicated by blue arrows do not match a calculated data point, these plateaus are considered as partial pathways connecting to one of the existing efficient conductive paths.

Initially when the number of efficient junctions is low the probability of observing the formation of a new EPP is low. As annealing continues more junctions are sintered and the chance to form an EPP increases. After the formation of the first efficient percolating path it is expected that the efficient junction density is sufficiently high that other pathways also begin to form in a more and more rapid succession. When plotted in the time domain (Fig. 3b) this should be seen as the length of plateaus decreasing on average as the number of plateaus increases. However this is a statistical process so the length of the plateaus are somewhat random, hence the experimental variability of the plateau length. As the number of EPP increases it becomes increasingly likely that new efficient paths overlap with existing paths, thus as $n$ increases so too does the probability of partial path formation, this results in the loss of quantized steps in $R(t, T)$ as the number of plateaus increases and a continuous drop in resistance is eventually observed (typically for values lower than $1000 \Omega$ ) for long annealing or at higher temperature.
Let us finally consider that while stick percolation is generally a density problem, in the present case the model works at a constant stick density upon which a network of junction resistances is applied which play the role of the percolation sites. Each site is a given resistance which is reduced by thermal annealing; conduction through the system will occur predominantly through low resistance junctions. These junctions represent a very large population (measured experimentally as about $10^{7} \mathrm{~cm}^{-2}$ for a specimen with similar density to that shown in Fig. 1a) with a large distribution of junction resistances. In the as-deposited state the majority of the junctions have a high electrical resistance. By heating, some of these junctions become more conductive. As the annealing proceeds, clusters of nanowires are connected by efficiently conducting junctions. When a large enough number of junction resistances are reduced, an efficient percolating pathway is formed spanning the network resulting in the sudden drops of the resistance reported in Fig. 3. The sudden electrical resistance drops observed in Fig. 3 can therefore be considered as quantized activation of percolation.

\subsection{Pre-annealing}

Another way of studying the effects of thermal annealing is to perform a pre-annealing at low temperature (in the present paper, a pre-annealing is considered as an isothermal anneal performed after the AgNW network fabrication and before a continuous ramp annealing). The effects of such pre-annealing on an experiment similar to the one reported in Fig. 1 (i.e. thermal ramp) are shown on Fig. 4. Pre-annealing was performed at $100{ }^{\circ} \mathrm{C}, 150{ }^{\circ} \mathrm{C}$ and $200{ }^{\circ} \mathrm{C}$ for 8,6 and 2 hours, respectively. The appropriate duration of each pre-anneal was determined by monitoring in situ the resistance of the network: when the resistance became stable the pre-anneal

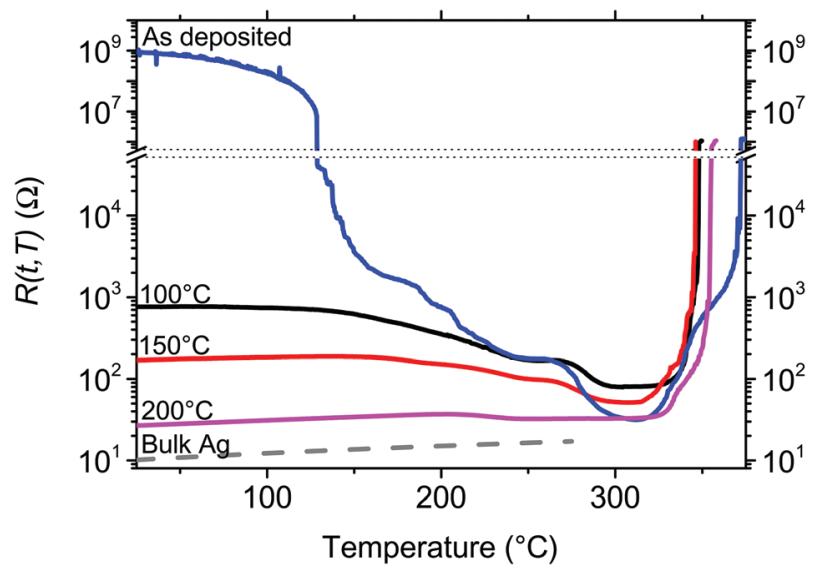

Fig. 4 Temperature dependence of the electrical resistance $R(t, T)$ of silver nanowire networks (concentration of $0.75 \mathrm{mg} \mathrm{mL}^{-1}$ ) during a ramp thermal annealing of $15{ }^{\circ} \mathrm{C}$ per minute for 4 different samples: as-deposited and pre-annealed at $100{ }^{\circ} \mathrm{C}, 150{ }^{\circ} \mathrm{C}$ and $200{ }^{\circ} \mathrm{C}$. The dashed curve is associated with the temperature-dependence of bulk silver resistivity, showing a good agreement between experimental data and phonon-induced increase of $R(t, T)$ up to $200{ }^{\circ} \mathrm{C}$ for the $200{ }^{\circ} \mathrm{C}$ preannealed specimen. 
was considered to have reached completion. This allows the exploration of temperature ranges over which different mechanisms are active.

The effects of the pre-annealing is drastic for the $R(t, T)$ dependence in the low temperature range (i.e. below $270{ }^{\circ} \mathrm{C}$ ). There is a continuous effect of pre-annealing for which a higher associated temperature corresponds to a lower $R(t, T)$ value in the low temperature range. Then for the higher temperature range the $R(t, T)$ behavior remains similar for all specimens: the pre-annealing does not appear to influence the onset of the NW instability.

Note that for the pre-annealing at $200{ }^{\circ} \mathrm{C}$, the increase of the $R(t, T)$ versus temperature (below $200{ }^{\circ} \mathrm{C}$ ) corresponds rather well to the increase of the electrical resistivity of bulk $\mathrm{Ag}$ associated with the scattering of electrons by the phonon population. This corresponds to the resistivity temperature dependence:

$$
\rho\left(T_{0}+\Delta T\right)=\rho_{0}\left(T_{0}\right) \cdot(1+\beta \cdot \Delta T)
$$

where $\beta$ is equal to $2.8 \times 10^{-3} \mathrm{~K}^{-1}$ for bulk silver. ${ }^{34}$ Such dependency is shown as the dashed line in Fig. 4: the experimental slope of the $200{ }^{\circ} \mathrm{C}$ preannealed AgNW network is close to the one calculated with the bulk silver $\beta$ value (although such a simple calculation does not consider the junction resistances between adjacent NWs).

\subsection{Physical mechanisms at play during thermal annealing}

We discuss now the relevant physical mechanisms at play for the evolution of the networks electrical properties during thermal annealing. These mechanisms are: solvent desorption, PVP desorption, sintering at contacts between adjacent nanowires and spheroidization. The first three mechanisms act to decrease the electrical resistance of the network while the last one leads to a drastic increase of $R(t, T)$. We will discuss these four mechanisms and their impact on the experimental results presented above.

The prevailing mechanism at play at low temperature (30-140 ${ }^{\circ} \mathrm{C}$ ) is desorption. At first, physical desorption of organic residues is responsible. Organic residues in the form of a very thin shell as visible in Fig. 1b are present between AgNWs due to capillary effects and act as electrical barriers, resulting in a high electrical resistance. The physical desorption of these residues enables a more intimate contact between NWs reducing the electrical resistance. After a preannealing at $100{ }^{\circ} \mathrm{C}$ for 8 hours is performed, the $R(t, T)$ curve exhibits a continuous behavior (no more sudden drops are observed) and $R(t, T)$ values are much smaller, indicating that there are already a large number of efficient conduction paths through the network. At $150{ }^{\circ} \mathrm{C}$ the glass transition temperature of PVP is reached, the thin shell of PVP coating the AgNWs $(\approx 5 \mathrm{~nm})$ begins to flow; this allows a more intimate contact between adjacent AgNWs, also decreasing the electrical resistance. At higher temperatures (150-250 ${ }^{\circ} \mathrm{C}$ ) the PVP undergoes thermal degradation and is completely removed from the surface of the wires (at least in air while different behavior can be observed under vacuum $\left.{ }^{35}\right)$. The reduction in $R(t, T)$ observed from $200-250{ }^{\circ} \mathrm{C}$ in Fig. 1f originates from the onset of local sintering, as indicated by the SEM observations (see Fig. 1c and d). It is important to note that for ramped annealing as shown in Fig. 1a and 4 this drop in $R(t, T)$ due to sintering is observed at higher temperature $\left(275-300{ }^{\circ} \mathrm{C}\right)$. This difference is likely due to the kinetics of the system. As both the experiments depicted by Fig. 1a and 4 are performed as thermal ramps at $15{ }^{\circ} \mathrm{C} \mathrm{min}^{-1}$ there is insufficient time for all of the PVP and organic residues to be removed in the temperature range $200-250{ }^{\circ} \mathrm{C}$, which results in a delay of the sintering. The preannealing shown in Fig. 4 allows some separation of the kinetics and critical temperatures of various mechanisms. Comparing the $R(t, T)$ of the as deposited sample to the initial resistance of the preannealed samples reveals that for each of the preanneal treatments the initial resistance corresponds closely to a plateau in the as-deposited $R(t, T)$. These plateaus result from a kinetic pinning of the onset of different mechanisms; as the samples are exposed to a thermal ramp the temperature continues to increase before each mechanism has reached completion. By performing a preanneal until the resistance stabilizes at different temperatures we reveal what the final resistance of the network obtained is once the mechanisms activated at the given temperature reach completion. This leads to the observation that sintering processes can reach completion at a temperature as low as $200{ }^{\circ} \mathrm{C}$ if sufficient time is provided. Sintering is the term usually used to describe the increase in mechanical strength and decrease in porosity of powder particle network when heated at a temperature approaching, but below, its melting point. ${ }^{36,37}$

In the present study, as the sintering mechanism concerns only the contacts between adjacent NWs, this process is called local sintering. This corresponds to the initial stages of the usual sintering phenomenon in powder metallurgy. The associated driving force for sintering is the reduction of surface energy. In general the smaller the particle size the lower the temperature where sintering will be observed. For instance the investigation of the sintering effect on the morphology and conductivity of $\mathrm{Ag}$ particles of $40 \mathrm{~nm}$ in diameter showed that sintering occurred at $100{ }^{\circ} \mathrm{C}$ and probably even below, ${ }^{38}$ while the melting point of silver is $962{ }^{\circ} \mathrm{C} .{ }^{34}$ In the case of AgNWs, sintering appears to be nearly complete after a pre-annealing at $200{ }^{\circ} \mathrm{C}$ for 2 hours since no further $R(t, T)$ decrease is observed as shown in Fig. 4 (as compared to the specimens pre-annealed at $150{ }^{\circ} \mathrm{C}$ or below).

\subsection{Spheroidization}

When submitted to a thermal annealing, nanowires can fragment into a chain of nanospheres above a certain temperature. ${ }^{39,40}$ This well-known morphological instability is often called Rayleigh or Plateau-Rayleigh instability and has been the subject of many investigations since its first study in the nineteenth century. ${ }^{41,42}$ Due to the major role played by metallic and semi-conductor nanowires in nanoelectronics and optoelectronics, ${ }^{1}$ the morphological Rayleigh instabilities of these materials have been investigated in the past. Studies of 
the role of wire diameter, temperature and annealing on the nanowire morphology evolution have already been reported. ${ }^{39,40,43}$ The physical origin of the Rayleigh instability is also the reduction of the surface energy. If one considers a liquid cylinder with initial radius $r$ and a sinusoidal perturbation along its axis, i.e.

$$
r(x)=r_{0}(\varepsilon)+\varepsilon \cdot \sin \left(\frac{2 \pi x}{\lambda}\right)
$$

simple calculations show that the perturbations with wavelength $\lambda>2 \pi r_{o}$ will increase spontaneously in amplitude and thus lead to a fragmentation of the initial cylinder. ${ }^{44}$ In reality, the morphological instabilities of a solid wire are slightly more complicated but the overall trend that is experimentally observed still remains the same. ${ }^{15,16,45}$ For instance the average distance between Ag nanoparticles in Fig. 1e is about $750 \mathrm{~nm}$, a value larger but of the same order of magnitude as $2 \pi R(330 \mathrm{~nm})$ (the difference stemming from the substrate influence, the fivefold twin structure of the wire as well coalescence which occurs after spheroidization). The Rayleigh criterion $\left(\lambda>2 \pi r_{\mathrm{o}}\right)$ is valid for all wire sizes, but since nanowires exhibit much higher surface to volume ratio the associated kinetics are much faster for nanowires with small diameters. The origin of this diameter-dependent kinetics stems from the dependence of the Rayleigh instability on surface diffusion. Smaller diameter wires have a higher ratio of surface to volume and a lower total volume. This means less atoms are required to move before spheroidization occurs and a higher proportion of the atoms are moving at a given time. This morphological instability leads to a loss of the percolation character of the network and hence a sharp increase of the electrical resistance at high temperature as observed in Fig. 1 and 4. Fig. 5 demonstrates that the diameter of the wires is an important factor for the onset of the Rayleigh instability,

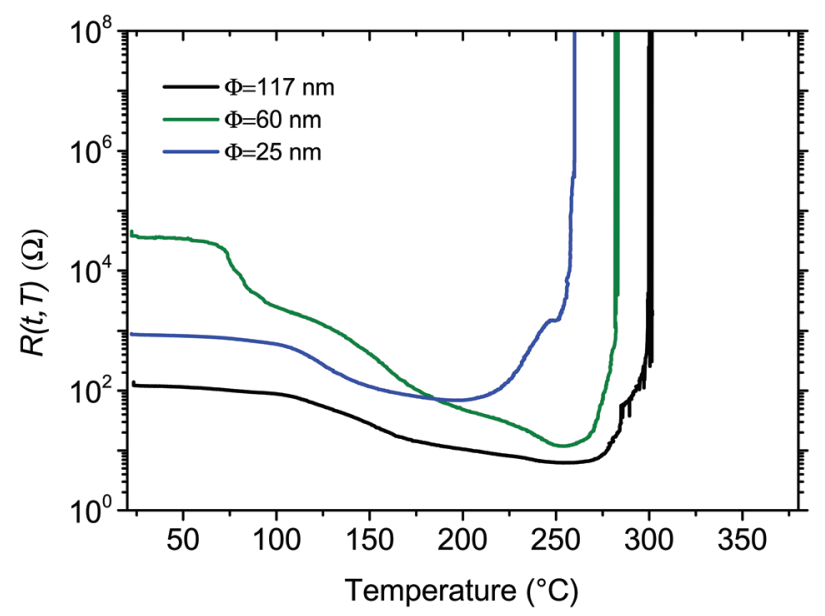

Fig. 5 The impact of diameter on the response of AgNW networks to temperature when thermal ramps are used from room temperature at a rate of $2{ }^{\circ} \mathrm{C}$ per minute. The three AgNW networks have similar areal mass density of $\approx 50 \mathrm{mg} \mathrm{m}^{-2}$. as the wire diameter decreases so does the temperature of spheroidization.

Knowing that in order to improve the electro-optical properties of AgNW networks, NWs with small diameter and large aspect ratio are often preferred, ${ }^{3}$ underlines the significance of the Rayleigh instability which becomes more of a limitation when the NW diameter decreases.

Finally a rather direct comparison can be outlined between the present work and the investigation of Chou et al. ${ }^{38}$ who studied the thermal effects on the properties of a film composed of Ag nanoparticles (also surrounded by a PVP layer). In both studies, in spite of the large difference in the morphology of the $\mathrm{Ag}$ nanostructures, the electrical resistance of the network exhibits a minimum versus the annealing temperature. In the study of Chou and co-authors, sintering and/or desorption of PVP lead to a decrease of $R(t, T)$ at low temperatures $50-200{ }^{\circ} \mathrm{C}$, while above $200-250{ }^{\circ} \mathrm{C}$ (depending on the nanoparticle size), $R(t, T)$ increased and the networks lose their conductive properties above $400{ }^{\circ} \mathrm{C}$ due to the growth of both $\mathrm{Ag}$ particles and pores and the eventual breakdown of conduction pathways. These observations on spherical nanoparticles by Chou et al. are partially analogous to the thermal behavior presented here for $\mathrm{Ag}$ nanowires.

\section{Conclusions}

This work demonstrates that thermal annealing can drastically lower the electrical resistance of AgNW network post deposition. The electrical resistance of the networks exhibit a minimum for an annealing temperature spanning from 200 to $320^{\circ} \mathrm{C}$. Four physical mechanisms have been identified at the origin of this resistance variation with temperature: desorption of organic residues, desorption/degradation of PVP, local sintering and Rayleigh instability of the NWs. All these mechanisms were discussed and their effects described. Such an investigation enables the determination of the thermal treatment required to produce the minimum electrical resistance for a given NW density.

Annealing an AgNW network fabricated with wires of $105 \mathrm{~nm}$ in diameter and $37.5 \mu \mathrm{m}$ in length at $200{ }^{\circ} \mathrm{C}$ for 2 hours results in a reduction in the sheet resistance from approximately $10^{7} \Omega \mathrm{sq}^{-1}$ to $9.5 \Omega \mathrm{sq}^{-1}$ associated with an optical transmission of $90 \%$ at $550 \mathrm{~nm}$ (once the substrate transmission is removed) resulting in state-of-the-art transparent electrodes. ${ }^{22}$ Moreover sudden drops of the networks' electrical resistance were observed and interpreted as the creation of efficient percolating pathways through the network. A simple model was proposed and describes well the experimental observations. Based on the results of the present research, there are two important directions that future investigations should focus on. In order to improve the thermal stability of nanowires, embedding them within a thin oxide layer could provide an efficient method to prevent morphological instabilities. This idea has already been considered with thin transparent oxide films $\left(\mathrm{ZnO}\right.$ or $\left.\mathrm{TiO}_{2}\right)$ for which slightly higher 
thermal stability was achieved..$^{25}$ Finally the quantitative estimation of the different kinetics involved for each physical mechanism would enable better separation of their relative influences and would help calculating their importance during thermal annealing. This could yield an efficient way to foresee the evolution of the morphological and electrical properties of metallic NW networks as induced by thermal annealing and allow further optimization of the physical properties of metallic NW networks.

\section{Experimental section}

AgNWs (Ag115, Ag60 and Ag25 in isopropanol) were purchased from SeaShell Technology. ${ }^{46}$ These nanowires were obtained from the polyol process ${ }^{32,47,48}$ for which NWs are grown in solution and are stabilized by polyvinylpyrrolidone (PVP). The nanowires are crystalline and present the five fold twinned structure typical for silver nanowires produced by the polyol method. $^{28,49}$ This solution was then diluted with isopropyl alcohol (IPA) to various concentrations.

Substrates used were Corning C1737-S111, alkaline earth boro-aluminosilicate glass, typical RMS roughness as measured by AFM was $3.4 \mathrm{~nm}$ with a maximum RMS roughness of $4 \mathrm{~nm}$. Before spin coating the AgNW solution the substrates were cleaned by sonication in acetone for 5 minutes followed by boiling in Isopropanol for 15 minutes and then drying with nitrogen.

Deposition was performed at room temperature by spincoating at $1500 \mathrm{rpm}$ as follows: $1 \mathrm{~mL}$ of solution was introduced onto the rotating substrate $(25 \mathrm{~mm} \times 25 \mathrm{~mm}$ corning glass, Corning 1737) in a drop-wise manner of two stages ( $0.5 \mathrm{~mL}$ each) with a $30 \mathrm{~s}$ pause between each deposition. The sample was maintained at a constant rotation throughout the deposition. This produces random networks of AgNWs on glass substrates.

Square specimens of $12 \mathrm{~mm} \times 12 \mathrm{~mm}$ were cut from the larger substrates and Ag paint electrodes were deposited on two opposite edges of the network for in situ measurement of the electrical resistance of the AgNW network during thermal annealing. This was performed with a two-point probe system where the electrical resistance was recorded using a Keithley sourcemeter (model 2400). Temperature is PID-controlled and recorded via two thermocouples mounted inside the heating stage and on the surface on which the sample rests, respectively.

Structural observations of the AgNWs were performed using a field-emission scanning electron microscopy (FESEM) ZEISS Ultra Plus microscope using an accelerating voltage of $10 \mathrm{kV}$. Any annealing or pre-annealing was performed in air.

\section{Acknowledgements}

This work has been supported by the Agence Nationale de la Recherche (Programme Matériaux et Procédés) through the project FICHTRE and by the project SEI from Grenoble INP. This work was also supported by the EACEA Erasmus Mundus funding program through IDS-FunMats. The authors would like to warmly thank J.P. Simonato, C. Celle, and C. Mayousse for fruitful discussions as well as M. Anikin and M. Jouvert for their assistance during experimental measurements.

\section{References}

1 J. Ji, Z. Zhou, X. Yang, W. Zhang, S. Sang and P. Li, Small, 2013, 9, 3014-3029.

2 S. De, P. J. King, P. E. Lyons, U. Khan and J. N. Coleman, ACS Nano, 2010, 4, 7064-7072.

3 S. Sorel, P. E. Lyons, S. De, J. C. Dickerson and J. N. Coleman, Nanotechnology, 2012, 23, 185201.

4 T. Tokuno, M. Nogi, M. Karakawa, J. Jiu, T. T. Nge, Y. Aso and K. Suganuma, Nano Res., 2011, 4, 1215-1222.

5 E. C. Garnett, W. Cai, J. J. Cha, F. Mahmood, S. T. Connor, M. Greyson Christoforo, Y. Cui, M. D. McGehee and M. L. Brongersma, Nat. Mater., 2012, 11, 241-249.

6 J. Lee, P. Lee, H. Lee, D. Lee, S. S. Lee and S. H. Ko, Nanoscale, 2012, 4, 6408-6414.

7 S. Han, S. Hong, J. Ham, J. Yeo, J. Lee, B. Kang, P. Lee, J. Kwon, S. S. Lee, M.-Y. Yang and S. H. Ko, Adv. Mater., 2014, 26, 5808-5814.

8 J.-Y. Lee, S. T. Connor, Y. Cui and P. Peumans, Nano Lett., 2008, 8, 689-692.

9 L. Hu, H. S. Kim, J.-Y. Lee, P. Peumans and Y. Cui, ACS Nano, 2010, 4, 2955-2963.

10 S. B. Sepulveda-Mora and S. G. Cloutier, J. Nanomater., 2012, 2012, 7.

11 G. Guisti, D. P. Langley, M. Lagrange, R. Collins, C. Jiménez, Y. Bréchet and D. Bellet, Int. J. Nanotechnol., 2014, 11, 785-795.

12 J. H. Lee, P. Lee, D. Lee, S. S. Lee and S. H. Ko, Cryst. Growth Des., 2012, 12, 5598-5605.

13 S. De, T. M. Higgins, P. E. Lyons, E. M. Doherty, P. N. Nirmalraj, W. J. Blau, J. J. Boland and J. N. Coleman, ACS Nano, 2009, 3, 1767-1774.

14 S. Coskun, E. Selen Ates and H. Emrah Unalan, Nanotechnology, 2013, 24, 125202.

15 H. S. Shin, J. Yu and J. Y. Song, Appl. Phys. Lett., 2007, 91, 173106-173108.

16 H. Li, J. M. Biser, J. T. Perkins, S. Dutta, R. P. Vinci and H. M. Chan, J. Appl. Phys., 2008, 103, 024315-024323.

17 H. Wong, J. Appl. Phys., 2012, 111, 103509-103517.

18 A. Kim, Y. Won, K. Woo, C.-H. Kim and J. Moon, ACS Nano, 2013, 7, 1081-1091.

19 L. Kavan, M. Grätzel, J. Rathouský and A. Zukalb, J. Electrochem. Soc., 1996, 143, 394-400.

20 C.-C. Yang, H. Q. Zhang and Y. R. Zheng, Curr. Appl. Phys., 2011, 11, S147-S153.

21 H. H. Khaligh and I. A. Goldthorpe, Nanoscale Res. Lett., 2013, 8, 1-6. 
22 D. Langley, G. Giusti, C. Mayousse, C. Celle, D. Bellet and J.-P. Simonato, Nanotechnology, 2013, 24, 452001.

23 V. Scardaci, R. Coull, P. E. Lyons, D. Rickard and J. N. Coleman, Small, 2011, 7, 2621-2628.

24 J.-W. Lim, D.-Y. Cho, K. Eun, S.-H. Choa, S.-I. Na, J. Kim and H.-K. Kim, Sol. Energy Mater. Sol. Cells, 2012, 105, 69-76.

25 F. S. F. Morgenstern, D. Kabra, S. Massip, T. J. K. Brenner, P. E. Lyons, J. N. Coleman and R. H. Friend, Appl. Phys. Lett., 2011, 4, 183307.

26 J. Ajuria, I. Ugarte, W. Cambarau, I. Etxebarria, R. TenaZaera and R. Pacios, Sol. Energy Mater. Sol. Cells, 2012, 102, 148-152.

27 J. Liang, L. Li, X. Niu, Z. Yu and Q. Pei, Nat. Photonics, 2013, 7, 817-824.

28 P. Lee, J. Lee, H. Lee, J. Yeo, S. Hong, K. H. Nam, D. Lee, S. S. Lee and S. H. Ko, Adv. Mater., 2012, 24, 33263332.

29 C. Celle, C. Mayousse, E. Moreau, H. Basti, A. Carella and J.-P. Simonato, Nano Res., 2012, 5, 427-433.

30 S. Sorel, D. Bellet and J. N. Coleman, ACS Nano, 2014, 8, 4805-4814.

31 H. Ibach, Physics of surfaces and interfaces, Springer, Berlin, New York, 2006.

32 Y. Sun, B. Mayers, T. Herricks and Y. Xia, Nano Lett., 2003, 3, 955-960.

33 A. Bid, A. Bora and A. K. Raychaudhuri, Phys. Rev. B: Condens. Matter, 2006, 74, 035426.

34 D. R. Lide, CRC Handbook of Chemistry and Physics 20042005: A Ready-Reference Book of Chemical and Physical Data, CRC press, 2004.
35 A. Mayoral, L. F. Allard, D. Ferrer, R. Esparza and M. JoseYacaman, J. Mater. Chem., 2010, 21, 893-898.

36 R. M. German, Sintering Theory and Practice, Wiley, 1996.

37 A. Vagnon, O. Lame, D. Bouvard, M. D. Michiel, D. Bellet and G. Kapelski, Acta Mater., 2006, 54, 513-522.

38 K.-S. Chou, K.-C. Huang and H.-H. Lee, Nanotechnology, 2005, 16, 779-784.

39 M. E. Toimil Molares, A. G. Balogh, T. W. Cornelius, R. Neumann and C. Trautmann, Appl. Phys. Lett., 2004, 85, 53375339.

40 S. Karim, M. E. Toimil-Molares, A. G. Balogh, W. Ensinger, T. W. Cornelius, E. U. Khan and R. Neumann, Nanotechnology, 2006, 17, 5954-5959.

41 J. Plateau, Translated Annual Reports of the Smithsonian Institution, 1873, 1863.

42 L. Rayleigh, Proc. London Math. Soc., 1878, s1-10, 4-13.

43 C.-P. Hsiung, H.-W. Liao, J.-Y. Gan, T.-B. Wu, J.-C. Hwang, F. Chen and M.-J. Tsai, ACS Nano, 2010, 4, 5414-5420.

44 P.-G. de Gennes, F. Brochard-Wyart and D. Quéré, Capillarity and Wetting Phenomena-Drops, Bubbles, Pearls, Waves, Springer, New York, 2004.

45 J. P. Naik, K. Das, P. D. Prewett, A. K. Raychaudhuri and Y. Chen, Appl. Phys. Lett., 2012, 101, 163108.

46 http://www.seashelltech.com/index.shtml, http://www. seashelltech.com/index.shtml.

47 Y. Sun, B. Gates, B. Mayers and Y. Xia, Nano Lett., 2002, 2, 165-168.

48 Y. Sun and Y. Xia, Adv. Mater., 2002, 14, 833-837.

49 Y. Sun, Y. Ren, Y. Liu, J. Wen, J. S. Okasinski and D. J. Miller, Nat. Commun., 2012, 3, 971. 